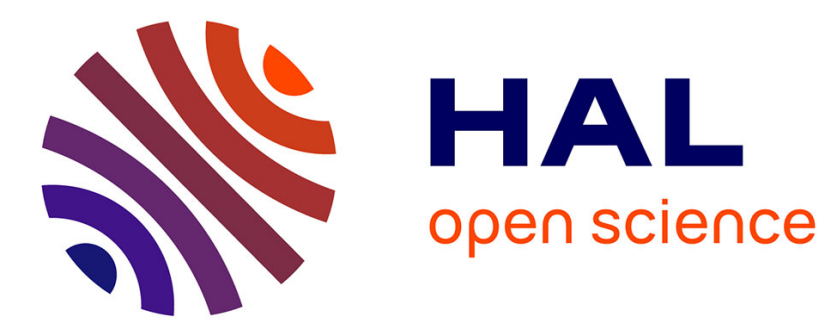

\title{
Diagramme d'équilibre tridimensionnel de la condensation des plasmas froids (Ta, A, O)* obtenus par pulvérisation cathodique réactive
}

\author{
R. Boistelle, G. Perny
}

\section{- To cite this version:}

R. Boistelle, G. Perny. Diagramme d'équilibre tridimensionnel de la condensation des plasmas froids (Ta, A, O)* obtenus par pulvérisation cathodique réactive. Revue de Physique Appliquée, 1968, 3 (2), pp.121-126. 10.1051/rphysap:0196800302012100 . jpa-00242835

HAL Id: jpa-00242835

https://hal.science/jpa-00242835

Submitted on 1 Jan 1968

HAL is a multi-disciplinary open access archive for the deposit and dissemination of scientific research documents, whether they are published or not. The documents may come from teaching and research institutions in France or abroad, or from public or private research centers.
L'archive ouverte pluridisciplinaire HAL, est destinée au dépôt et à la diffusion de documents scientifiques de niveau recherche, publiés ou non, émanant des établissements d'enseignement et de recherche français ou étrangers, des laboratoires publics ou privés. 


\title{
DIAGRAMME D'ÉQUILIBRE TRIDIMENSIONNEL DE LA GONDENSATION DES PLASMAS FROIDS (Ta, A, O)* OBTENUS PAR PULVÉRISATION GATHODIQUE RÉAGTIVE
}

\author{
Par R. BOISTELLE $\left({ }^{\mathbf{1}}\right)$ et G. PERNY, \\ Laboratoire de Physico-Chimie des Couches Minces, \\ École Supérieure de Chimie, 3, rue Werner, 68-Mulhouse.
}

(Reçu le 13 novembre 1967.)

\begin{abstract}
Résumé. - On étudie la vitesse de formation des couches minces de $\mathrm{Ta}$ et $\mathrm{Ta}_{2} \mathrm{O}_{5}$, obtenues par pulvérisation cathodique réactive, en fonction d'un certain nombre de paramètres expérimentaux (pression du ou des gaz, tension de pulvérisation, distance cathode-support...). On montre qu'il existe un ensemble de valeurs optimales de ces paramètres permettant l'obtention des couches minces de manière parfaitement reproductible. On aboutit à un diagramme d'équilibre tridimensionnel où à chaque point figuratif de l'espace correspond une couche mince d'épaisseur et de nature chimique bien déterminées. La microdiffraction et la microscopie électronique montrent respectivement que les couches minces sont quasi amorphes et que le diamètre moyen des cristallites est de l'ordre de $20 \AA$.
\end{abstract}

Abstract. - The deposition rate of thin films of $\mathrm{Ta}$ and $\mathrm{Ta}_{2} \mathrm{O}_{5}$ obtained by reactive cathodic pulverisation is studied in terms of experimental parameters (gas pressure, pulverisation voltage, cathode-substrate distance...). Optimal values of these parameters giving perfectly reproductible thin films are found. One obtains a tridimensional diagram in which each figurative point of the space corresponds to a thin film of well defined thickness and chemical composition. Electron microdiffraction and microscopy respectively show that the thin films are quasi amorphous and that the mean diameter of the crystallites is about $20 \AA$.

I. Introduction. - Nous exposons ici une étude effectuée dans le double but de montrer l'influence de certains paramètres expérimentaux sur l'obtention des couches minces de $\mathrm{Ta}$ et $\mathrm{Ta}_{2} \mathrm{O}_{5}$ et de trouver un ensemble de paramètres permettant de préparer ces couches de manière reproductible. Cette étude fondamentale doit entrer ultérieurement dans le cadre général d'une théorie de la pulvérisation cathodique réactive [1].

Le montage expérimental que nous avons utilisé a déjà été décrit par ailleurs [2], [3]. Signalons simplement ici que la cathode de tantale pur est d'un diamètre de $30 \mathrm{~mm}$ et que les couches de Ta et $\mathrm{Ta}_{2} \mathrm{O}_{5}$ ont été condensées soit dans les mélanges argonoxygène, soit dans ces gaz purs, sur des supports solides en silice.

(1) Laboratoire de Minéralogie-Cristallographie, Laboratoire des Mécanismes de la Croissance Cristalline, associé au C.N.R.S. ; Faculté des Sciences Saint-Jérôme, 13-Marseille $\left(13^{\mathrm{e}}\right)$.
Pour une géométrie de l'enceinte et un gaz ou mélange gazeux donnés, la vitesse de condensation du plasma froid créé dans le dispositif dépend essentiellement de trois paramètres expérimentaux : la tension de pulvérisation $V_{\mathrm{p}}$, la pression $p$ du ou des gaz et la distance $d$ de la cathode à la surface du substrat de condensation. A ceci s'ajoutent deux paramètres secondaires : la distance $\delta$ de la cathode à l'anode et la durée $t$ de la condensation, si celle-ci s'effectue, comme dans notre cas, en régime quasi statique. Quant à la nature chimique de la couche mince déposée, celle-ci dépend simultanément des paramètres précédents et de la composition du milieu gazeux ambiant [4], [5].

Si $\eta$ est la vitesse de condensation, on a :

$$
\eta=f\left(V_{p}, p, d, \delta, t\right)
$$

avec :

$$
V_{\mathrm{p}}=f\left(V_{\mathrm{a}}, p, \delta\right)
$$

où $V_{\mathrm{a}}$ est la tension appliquée. 

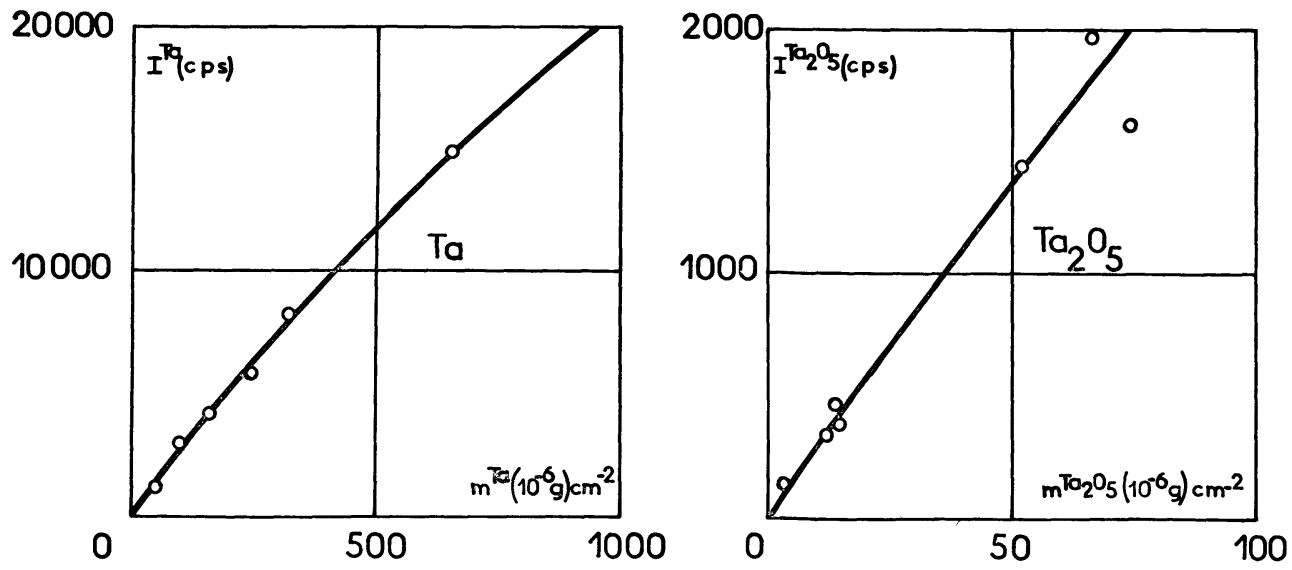

FIG. 1. - Intensités de fluorescence $\mathrm{X}$ en fonction des masses de $\mathrm{Ta}$ et $\mathrm{Ta}_{2} \mathrm{O}_{5}$.

Eu égard à la dureté des couches minces de Ta et $\mathrm{Ta}_{2} \mathrm{O}_{5}$ et à leur forte adhérence sur les supports $\mathrm{SiO}_{2}$, nous n'avons pas pu mesurer leurs épaisseurs de façon suffisamment précise pour pouvoir en déduire $\eta$. C'est pourquoi nous avons utilisé la méthode des comptages en fluorescence X (sur la raie LA1-1 de l'élément Ta) par une mise en corrélation des intensités de fluorescence $\mathrm{X}$ et des masses des couches minces déposées. Les pesées ont été effectuées sur une microbalance précise à $\pm 5 \times 10^{-6} \mathrm{~g}$. La figure 1 représente les intensités de fluorescence émises par des couches de $\mathrm{Ta}$ et $\mathrm{Ta}_{2} \mathrm{O}_{5}$ en fonction de leur masse superficielle (g. $\left.\mathrm{cm}^{-2}\right)$.

Comme $\eta$ est fonction d'un nombre élevé de paramètres, il s'agit d'ajuster la majorité d'entre eux à des valeurs permettant d'obtenir une condensation optimale sur le support. La connaissance des propriétés physiques et chimiques des couches minces obtenues et des paramètres intervenant dans les processus de condensation permet alors d'établir un diagramme d'équilibre tridimensionnel où à chaque point figuratif de l'espace correspond une couche mince d'épaisseur et de nature chimique bien déterminées.

II. Influence des paramètres $p, d, \delta$ et $t$ sur $\eta$. Pour éliminer l'influence sur $\eta$ de la composition du gaz ambiant, nous nous sommes limités à l'étude de la variation de la vitesse de condensation des couches minces de Ta fabriquées dans $100 \%$ A. Afin d'obtenir des résultats comparables, nous avons fixé $V_{\mathrm{a}}$ à $3000 \mathrm{~V}$. L'anode solidaire du support $\mathrm{SiO}_{2}$ est située à $\delta=(d+6) \mathrm{mm}$. Les pressions que nous indiquons sont celles lues directement sur une jauge Pirani, sans corrections.

II.1. Dans l'étude de la fonction $\eta=f(d)$, le paramètre $p$ est constant et égal à $50 \mu$. Ceci entraîne $V_{\mathrm{p}}=2460 \mathrm{~V}$ et $i=6 \mathrm{~mA}$, $i$ étant l'intensité du courant pendant la condensation. Sur la figure 2, qui

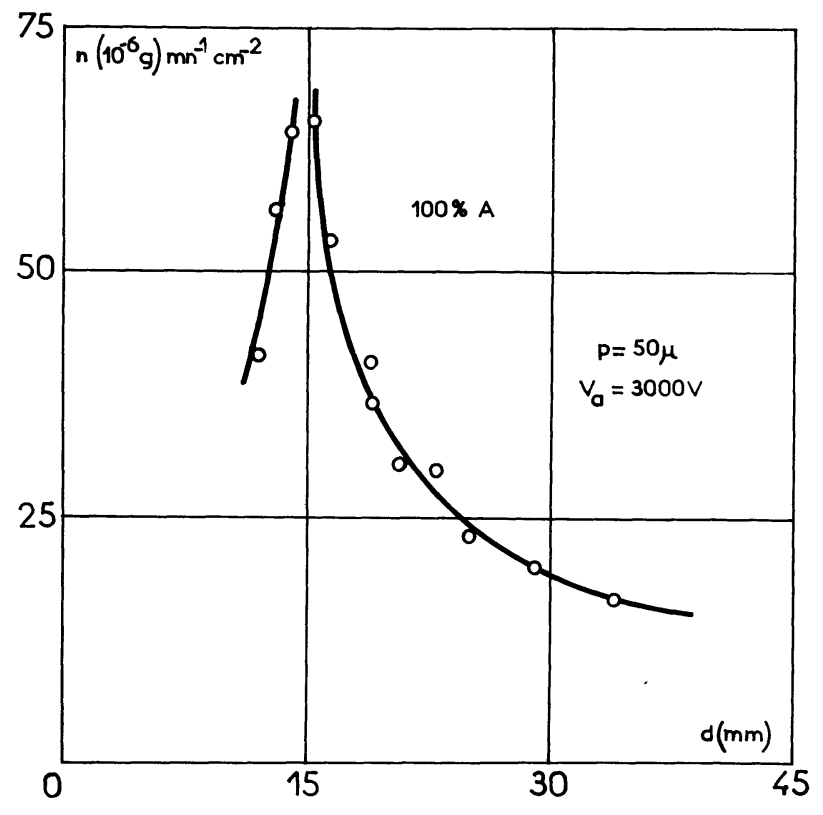

FIG. 2. - Vitesse de condensation en fonction de la distance de la cathode au support $\mathrm{SiO}_{2}$.

représente la vitesse de condensation $\eta$ par $\mathrm{cm}^{2} \mathrm{du}$ support $\mathrm{SiO}_{2}$, on remarque qu'il existe une valeur particulière de $d$ qui correspond approximativement à l'espace sombre de Crookes et au voisinage de laquelle $\eta$ atteint une valeur maximale. De part et d'autre de cette valeur particulière de $d, \eta$ diminue très vite.

II.2. L'influence sur $\eta$ du paramètre $p$ est plus complexe ( fig. 3) et pour la mettre en évidence nous avons maintenu $d$ à $24 \mathrm{~mm}$. Du point de vue expérimental, la fonction $\eta=f(p)$ n'est pas continue mais présente un point anguleux vers $p=40 \mu$ ainsi qu'un autre vers $p=30 \mu$. Ceci est à mettre en relation 


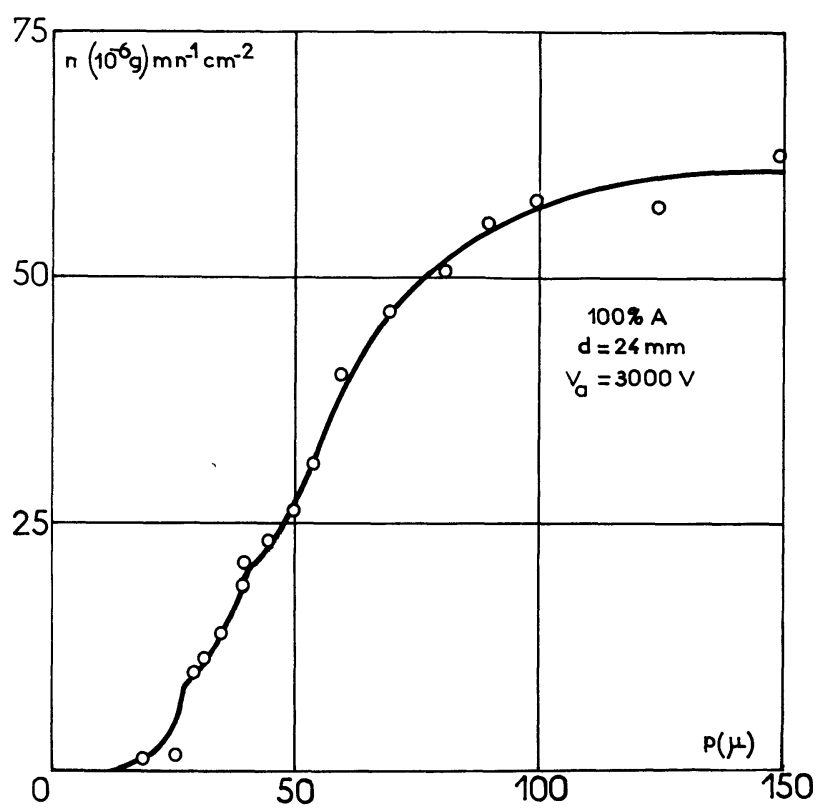

Fig. 3. - Vitesse de condensation en fonction de la pression d'argon.

Erratum. - En ordonnées, lire $\eta$.

avec les courbes de la figure 4 qui représentent respectivement la tension de pulvérisation $V_{\mathrm{p}}$ ( fig. $4 \mathrm{a}$ ) et l'intensité $i$ du courant ( fig. $4 \mathrm{~b}$ ) en fonction de $p$. Ces fonctions ne suivent une loi linéaire que pour $p>40 \mu$ et si l'on compare alors les figures 3 et 4 , on constate que c'est précisément vers $p=40 \mu$ que se situent les anomalies sur les trois courbes. Ces anomalies confirment le point anguleux à $p=40 \mu$ dans la fonction $\eta=f(p)$ qui ne semble donc devenir continue que pour $p>40 \mu$. Des réserves doivent être faites sur le point anguleux à $p=30 \mu$, mais il convient de dire que la lecture de $V_{\mathrm{p}}$ et de $i$ ne se fait qu'à $\pm 25 \mathrm{~V}$ et $\pm 0,5 \mathrm{~mA}$ près. Cette erreur est importante, car à une grande variation de $p(0-40 \mu)$ ne correspondent que de faibles variations de $V_{\mathrm{p}}(100 \mathrm{~V})$ et de $i(2,5 \mathrm{~mA})$.

II.3. Quant à la durée $t$ de la condensation, elle n'influe en rien sur les valeurs de $\eta$ lorsque le gaz ambiant est constitué de $100 \% \mathrm{~A}$ ou $100 \% \mathrm{O}_{2}$ (fig. 5). Les masses de $\mathrm{Ta}$ et $\mathrm{Ta}_{2} \mathrm{O}_{5}$ condensées varient linéairement avec $t$ dans un rapport $m_{\mathrm{Ta}^{2}} / m_{\mathrm{Ta}_{2} \mathrm{O}_{5}}$ voisin de 12.

Dans les mélanges $\mathrm{A}+\mathrm{O}_{2}$, par contre, et en régime quasi statique, la disparition progressive de

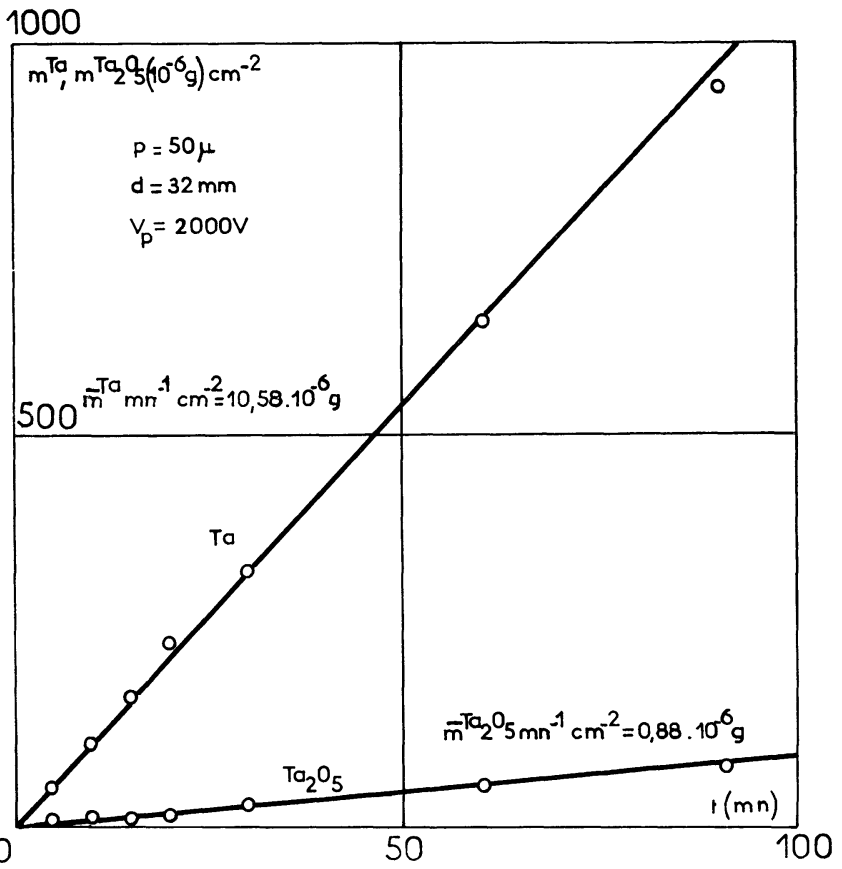

FIG. 5. - Masses de Ta et $\mathrm{Ta}_{2} \mathrm{O}_{5}$ déposées en fonction $\mathrm{du}$ temps de condensation.

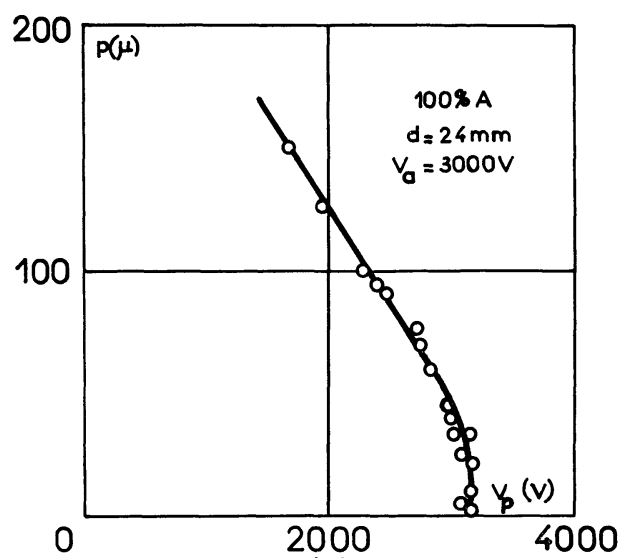

(a)

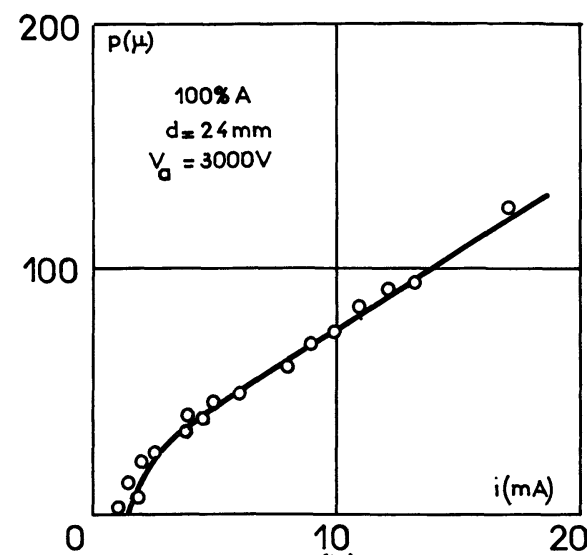

(b)

FIG. 4. - Tension de pulvérisation (a) et intensité du courant (b) en fonction de la pression d'argon. 


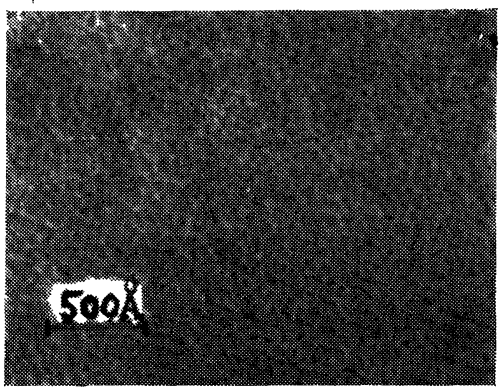

$t=1 / 2 \mathrm{mn}$

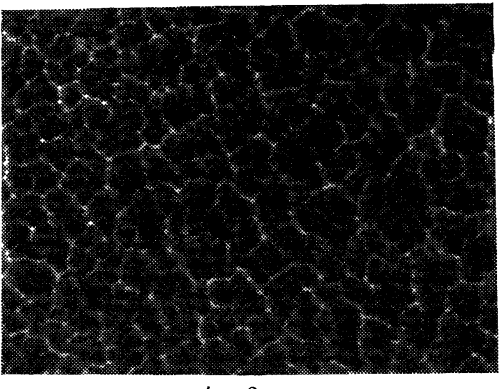

$t=2 \mathrm{mn}$

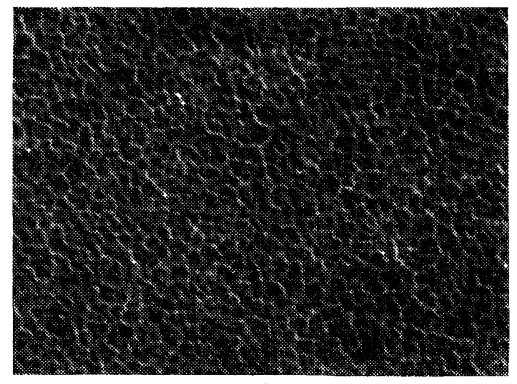

$t=1 \mathrm{mn}$

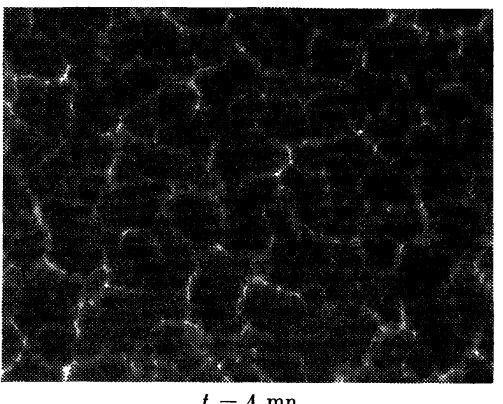

$t=4 \mathrm{mn}$

FIG. 7. - Aspect des couches minces de Ta en fonction de la durée de condensation.

l'oxygène influe de façon très sensible sur les vitesses de condensation (III.3). Il convient donc d'utiliser des durées de condensation suffisamment faibles pour que la concentration en oxygène du mélange gazeux puisse être considérée comme constante. C'est pourquoi nous avons choisi $t=15 \mathrm{mn}$ pour les gaz purs et $t=4 \mathrm{mn}$ pour les mélanges gazeux.

II.4. De l'étude précédente, nous avons tiré les conditions permettant d'obtenir des couches minces de tantale avec le maximum de précision et de reproductibilité $(d=32 \mathrm{mn}$ et $p=50 \mu)$. En effet, une erreur importante sur les paramètres $d$ et $p$ ( fig. 2 et 3 ) n'entraîne qu'une faible erreur sur la vitesse de condensation $\eta$. Comme $p, d, \delta$ et $t$ sont fixés, la vitesse de condensation peut alors être ramenée à son expression la plus simple $\eta=f\left(V_{\mathrm{p}}\right), \quad V_{\mathrm{p}}$ variant linéairement avec $V_{2}$.

Il convient de rappeler cependant que la relation ci-dessus n'est vraie que pour une géométrie de l'enceinte de condensation et une composition initiale du milieu gazeux données. C'est pourquoi le diagramme d'équilibre de la condensation des plasmas froids $(\mathrm{Ta}, \mathrm{A}, \mathrm{O})^{*}$ aura pour pôles : vitesse de condensation, tension de pulvérisation et concentration en oxygène du milieu ambiant.

III. Diagramme d'équilibre tridimensionnel. Quatre diagrammes plans $\eta=f\left(V_{\mathrm{p}}\right)$ correspondant à quatre mélanges différents de gaz ont été établis. Les vitesses $\eta$ ont été calculées à partir des données des figures 1 et 5 , et la nature chimique des couches minces a été déterminée par microdiffraction électronique.
III.1. Diagramme $\eta=f\left(V_{\mathrm{p}}\right)$ Pour $100 \% \mathrm{~A}($ fig. 6$)$. - La vitesse de condensation $\eta$ croît linéairement avec $V_{\mathrm{p}}$ au-dessus d'un certain seuil de condensation $\left(V_{\mathrm{p}} \simeq 1000 \mathrm{~V}\right)$, seuil qui se retrouve dans les figures 8 , 9 et 10. Pour des valeurs inférieures, la vitesse de condensation est négligeable. A chaque point expérimental correspond une couche mince de tantale pur.

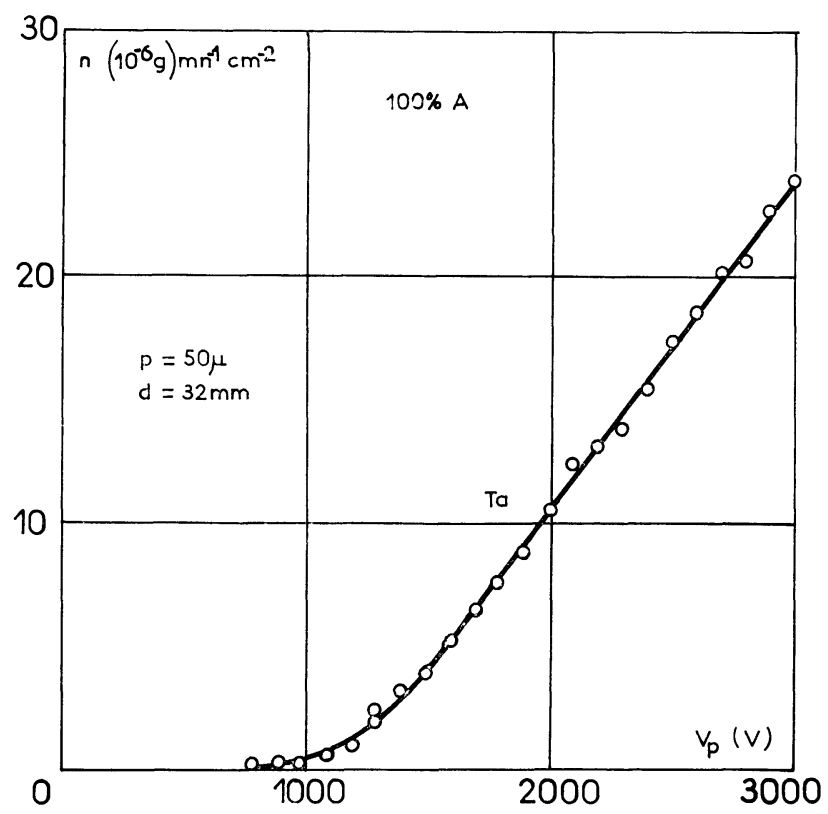

FrG. 6. - Vitesse de condensation de Ta dans $100 \% \mathrm{~A}$ en fonction de la tension de pulvérisation.

Erratum. - En ordonnées, lire $\eta$. 
Nous avons alors effectué une étude de la germination de $\mathrm{Ta}$ sur $\mathrm{Al}$ polycristallin à grains très fins (quelques dizaines d'angstrœms) dans des conditions expérimentales identiques $\left(V_{\mathrm{p}}=2000 \mathrm{~V}\right.$ constant $)$. La figure 7 montre 4 clichés de microscopie électronique par transmission correspondant aux durées de condensation $t=1 / 2,1,2$ et $4 \mathrm{mn}$ (grossissement $\times 200000$; $1 \mathrm{~cm}$ représente $500 \AA$ ). L'évolution du diamètre moyen $\varnothing$ des grains est nettement visible en fonction de $t$. Chacun de ces grains est constitué d'un agrégat de cristallites élémentaires de Ta, comme le prouvent les clichés de microdiffraction électronique qui présentent des raies très larges. D'après les clichés de microdiffraction et ceux de la microscopie, nous estimons que les dimensions des cristallites sont de l'ordre de $20 \AA$. Des échantillons recuits à $750^{\circ} \mathrm{C}$ donnent en microdiffraction des raies fines qui traduisent une recristallisation à l'intérieur des grains sans répercussion sur leur aspect extérieur. Les grains restent identiques après recuit à ceux de la figure 7 .

Dans le tableau ci-dessous, nous indiquons quelques valeurs concernant les clichés de la figure 7 .

\begin{tabular}{cccc}
$t(\mathrm{mn})$ & $m_{\mathrm{Ta}}\left(10^{-6} \mathrm{~g}\right) \mathrm{cm}^{-2}$ & $\varnothing(\AA)$ & $e(\AA)$ \\
\hline $1 / 2$ & - & - & - \\
1 & 5,29 & 50 & 32 \\
2 & 10,58 & 100 & 64 \\
4 & 21,16 & 200 & 128 \\
& 42,32 & 300 & 256
\end{tabular}

$m_{\mathrm{Ta}}=$ masse de Ta calculée à partir de la figure 5 ;

$\varnothing=$ diamètre moyen des grains observés sur la figure 7 .

$e=$ épaisseur évaluée en supposant une compacité égale à 1 ; il s'agit donc d'une épaisseur minimale

III.2. Diagramme $\eta=f\left(V_{\mathrm{p}}\right)$ Pour $100 \% \quad \mathrm{O}_{2}$ ( fig. 8). - La vitesse de condensation $\eta$ croît de plus en plus vite avec $V_{\mathrm{p}}$ jusqu'à $V_{\mathrm{p}}=2500 \mathrm{~V}$, valeur à partir de laquelle elle tend vers une limite supérieure. Ceci est à mettre en relation avec le fait que $V_{\mathrm{p}}$ ne varie plus linéairement avec $V_{\mathrm{a}}$ et qu'à une augmentation élevée de $V_{\mathrm{a}}$ ne correspond qu'une faible augmentation de $V_{\mathrm{p}}$. La microscopie révèle des grains ayant le même aspect que ceux de la figure 7 , alors que la microdiffraction d'échantillons recuits à $750^{\circ} \mathrm{C}$ indique que tous les points expérimentaux de la figure 8 représentent des couches minces de $\mathrm{Ta}_{2} \mathrm{O}_{5}$. Les échantillons non recuits ne donnent aucune raie nette de microdiffraction et nous en concluons que les couches minces de $\mathrm{Ta}_{2} \mathrm{O}_{5}$ obtenues sont quasi amorphes. Nous reportons ci-dessous quelques valeurs concernant ces couches minces pour différentes durées de condensation.

$\begin{array}{rrr}t(\mathrm{mn}) & m_{\mathrm{Ta}_{2} \mathrm{O}_{5}}\left(10^{-6} \mathrm{~g}\right) \mathrm{cm}^{-2} & \varnothing(\AA) \\ - & - & - \\ 3 & 2,64 & 25 \\ 5 & 4,40 & 50 \\ 10 & 8,80 & 50 \\ 20 & 17,60 & 150\end{array}$

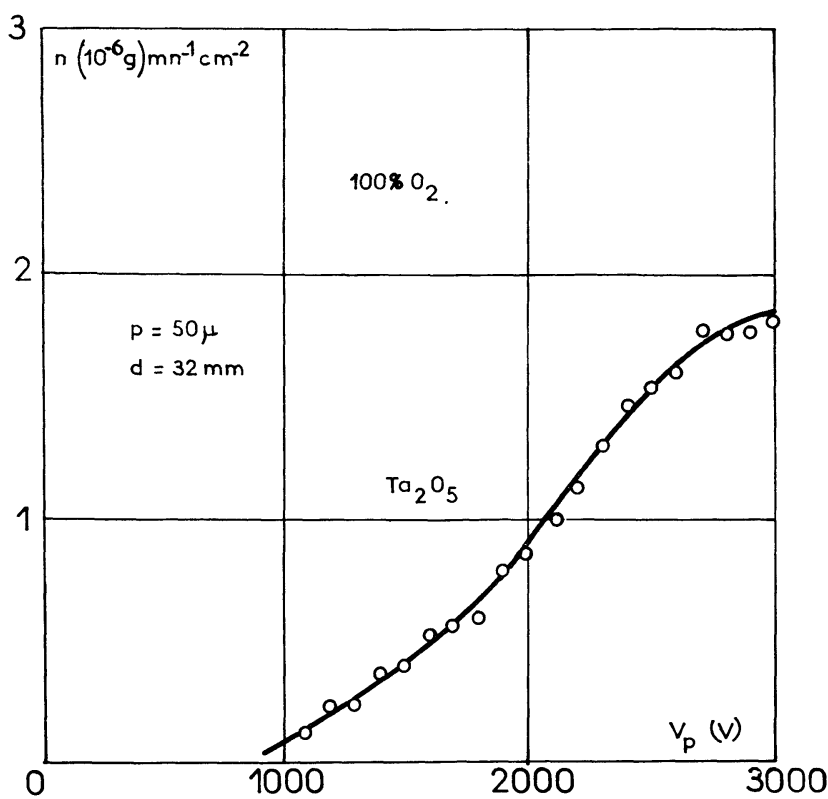

Frg. 8. - Vitesse de condensation de $\mathrm{Ta}_{2} \mathrm{O}_{5}$ dans $100 \% \mathrm{O}_{2}$ en fonction de la tension de pulvérisation.

Erratum. - En ordonnées, lire $\eta$.

III.3. Diagramme $\eta=f\left(V_{\mathrm{p}}\right)$ Pour $85 \% \mathrm{~A}+$ $15 \% \mathrm{O}_{2}$ (fig. 9). - Au-dessus du seuil de condensation, $\eta$ varie constamment avec $V_{p}$, la courbe ayant une pente moyenne plus forte que celle de la figure 8. A chaque point expérimental correspond une couche mince de $\mathrm{Ta}_{2} \mathrm{O}_{5}$.

Nous avons noté que, pour des durées de condensation supérieures à $4 \mathrm{mn}, \eta$ croît très vite et tend vers la même valeur que dans l'argon pur. G'est

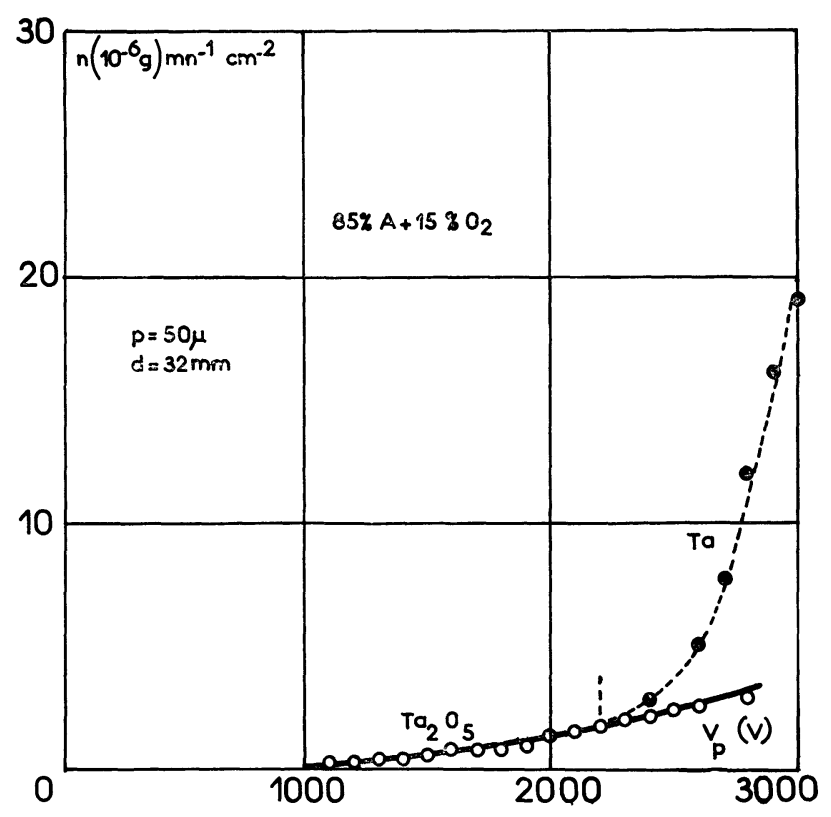

FIg. 9. - Vitesse de condensation de $\mathrm{Ta}_{2} \mathrm{O}_{5}$ dans $15 \% \mathrm{O}_{2}$ en fonction de la tension de pulvérisation.

Erratum. - En ordonnées, lire. $\eta$. 
pourquoi nous avons représenté par des cercles noirs (fig. 9) les résultats obtenus pour des durées $t$ de condensation de $15 \mathrm{mn}$. Il y a formation de $\mathrm{Ta}_{2} \mathrm{O}_{5}$ lorsque $1100<V_{\mathrm{p}}<2200 \mathrm{~V}$ et formation de Ta lorsque $V_{\mathrm{p}}>2200 \mathrm{~V}$ avec un domaine de transition $\mathrm{Ta}+\mathrm{Ta}_{2} \mathrm{O}_{5}$ au voisinage de $V_{\mathrm{p}}=2200 \mathrm{~V}$. Ceci illustre bien ce que nous disions plus haut, à savoir qu'en régime quasi statique la durée de condensation influe à la fois sur la vitesse de condensation et la nature chimique du dépôt.

III.4. Diagramme $\eta=f\left(V_{\mathrm{p}}\right)$ pour $70 \% \mathrm{~A}+$ $30 \% \quad \mathrm{O}_{2}$ (fig. 10). - La vitesse de consendation $\eta$ varie sensiblement de façon linéaire avec $V_{\mathrm{p}}$ jusqu'à $V_{\mathrm{p}}=2300 \mathrm{~V}$, valeur au-delà de laquelle la pente de

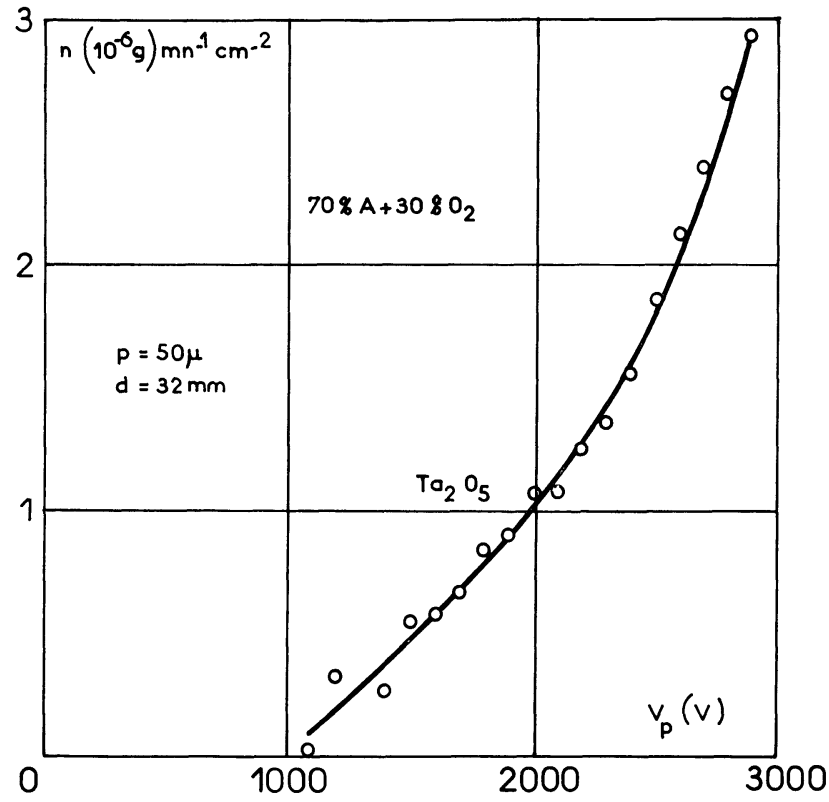

FIG. 10. - Vitesse de condensation de $\mathrm{Ta}_{2} \mathrm{O}_{5}$ dans $30 \% \mathrm{O}_{2}$ en fonction de la tension de pulvérisation.

Erratum. - En ordonnées, lire $\eta$.

la courbe augmente. La vitesse est à peine plus faible que dans le cas précédent (fig. 9) et chaque point correspond à la synthèse de couches minces de $\mathrm{Ta}_{2} \mathrm{O}_{5}$. Nous n'avons pas établi de diagrammes pour des concentrations en oxygène supérieures à $30 \%$, car à cette concentration déjà les valeurs de $\eta$ sont très voisines de celles à $100 \% \mathrm{O}_{2}$.

III.5. La mise en corrélation des quatre diagrammes plans étudiés permet de construire le diagramme tridimensionnel de la figure 11 dont les trois axes sont représentatifs des variations des paramètres principaux de la pulvérisation cathodique réactive.

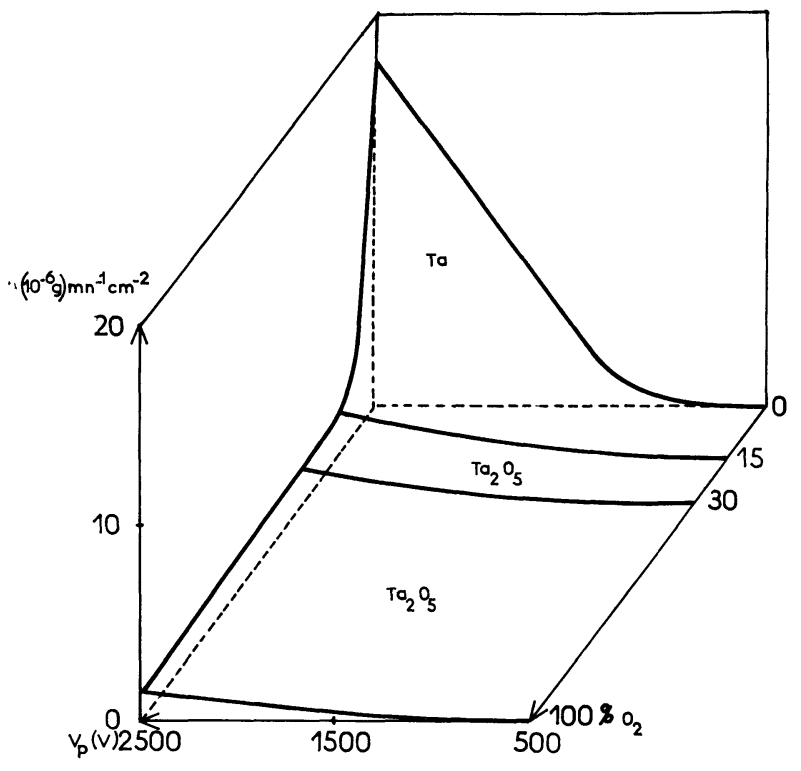

FIG. 11. - Diagramme d'équilibre tridimensionnel de la condensation des plasmas froids $(\mathrm{Ta}, \mathrm{A}, \mathrm{O})^{*}$ pour $d=32 \mathrm{mn}$ et $p=50 \mu$.

Erratum. - Suivant l'axe vertical, on porte $\eta$.

IV. Conclusion. - Nous avons examiné l'influence de la pression et de la distance cathode-support sur la vitesse de condensation des plasmas froids (Ta, $\mathrm{A}, \mathrm{O})$ * dans le but de trouver les conditions permettant d'obtenir des couches minces reproductibles de Ta et $\mathrm{Ta}_{2} \mathrm{O}_{5}$.

Nous avons élaboré quatre diagrammes représentant les variations de la vitesse de condensation en fonction de la tension de pulvérisation pour quatre mélanges argon-oxygène.

Les masses des couches minces ont été déterminées simultanément par pesée et fluorescence $\mathrm{X}$, leur nature chimique par microdiffraction électronique. Les diamètres des cristallites de $\mathrm{Ta}$ et $\mathrm{Ta}_{2} \mathrm{O}_{5}$ ont été évalués à environ $20 \AA$, les couches minces obtenues étant quasi amorphes. Un recuit à $750^{\circ} \mathrm{C}$ permet d'obtenir des recristallisations internes sans influence apparente sur la taille des grains.

Dans l'argon pur, on obtient des dépôts de Ta et dans l'oxygène des dépôts de $\mathrm{Ta}_{2} \mathrm{O}_{5}$. Dans les mélanges argon + oxygène se déposent soit Ta soit $\mathrm{Ta}_{2} \mathrm{O}_{5}$, d'une part selon la durée de la condensation, et d'autre part selon la tension appliquée.

L'ensemble des résultats peut être résumé sous forme de diagramme tridimensionnel où à chaque point figuratif de l'espace correspond une couche mince de masse et de nature chimique déterminées.

\section{BIBLIOGRAPHIE}

[1] Perny (G.), Samirant (M.), Lavitile Saint-Martin (B.), C.R. Acad. Sci., 1966, 262, 1841.

[2] Perny (G.), LaVilile SaINT-Martin (B.), RoesLER (P.), HALLER (B.), J. Physique, 1964, 25, 5.

[3] BOISTELIE (R.), Rapport D.R.M.E., 416/65/4, 1966.
[4] Perny (G.), Lavilile SaInT-Martin (B.), SamiRANT (M.), C.R. Acad. Sci., 1966, 263, 265.

[5] LAVII LE SAINT-MaRTIN (B.), PERNY (G.), SAMIRANT (M.), C.R. Acad. Sci., 1966, 263, 1181. 